\title{
First measurements of electron vorticity in the foreshock and solar wind
}

\author{
C. Gurgiolo ${ }^{1}$, M. L. Goldstein ${ }^{2}$, A. F. Viñas ${ }^{2}$, and A. N. Fazakerley ${ }^{3}$ \\ ${ }^{1}$ Bitterroot Basic Research, Hamilton, Montana, USA \\ ${ }^{2}$ Geospace Science Laboratory, Code 673, NASA Goddard Space Flight Center, Greenbelt, MD, USA \\ ${ }^{3}$ Mullard Space Science Laboratory, University College London, Holmbury St. Mary Dorking, Surrey RH5 6NT, UK
}

Received: 24 July 2010 - Revised: 8 December 2010 - Accepted: 9 December 2010 - Published: 21 December 2010

\begin{abstract}
We describe the methodology used to set up and compute spatial derivatives of the electron moments using data acquired by the Plasma Electron And Current Experiment (PEACE) from the four Cluster spacecraft. The results are used to investigate electron vorticity in the foreshock. We find that much of the measured vorticity, under nominal conditions, appears to be caused by changes in the flow direction of the return (either reflected or leakage from the magnetosheath) and strahl electron populations as they couple to changes in the magnetic field orientation. This in turn results in deflections in the total bulk velocity producing the measured vorticity.
\end{abstract}

Keywords. Interplanetary physics (Plasma waves and turbulence; Solar wind plasma; Instruments and techniques)

\section{Introduction}

Simply put, vorticity is the measure of rotation in a fluid flow. In a plasma, the presence of vorticity can, among other things, be an indication of turbulence, shear flow, or flow deflected around an object. The scale lengths over which the vorticity occurs is to a high degree a function of the source. Despite its usefulness, however, there have been only a few direct measurements of vorticity in space plasmas primarily due to the lack of near simultaneous multipoint measurements.

Direct observations of vorticity require the ability to make volumetric measurements which in turn requires simultaneous measurements at a minimum of four non-coplanar positions. Until the launch of Cluster this was not possible. Since then measurements of three-dimensional properties and symmetries of the magnetic fluctuations can and are being carried

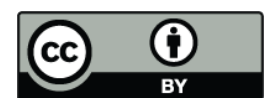

Correspondence to: C. Gurgiolo (chris@gurgiolo.com) out using data from the Fluxgate magnetometer (FGM) and Spatio-Temporal Analysis of Field Fluctuations (STAFF) experiments (see, e.g., Narita et al., 2006, 2010; Sahraoui et al., 2010; Fairfield et al., 2007) while PEACE data has been used to measure the divergence of the electron pressure tensor (Henderson et al., 2006, 2008) and electron vorticity in the magnetotail (Dewhurst, 2005). To date there have been no such attempts of computing spatial derivatives using plasma data in the upstream. The early failure of the Cluster Ion Spectrometry (CIS) Hot Ion Analyzer (HIA) experiment on two of the spacecraft left only the electron data from PEACE with the required minimum number of spatial measurement points.

Electrons, however, are often considered to provide a less than ideal fluid representation of the solar wind. This is most likely based on the recognition that for solar wind observations, electrons are subsonic, so that extracting accurate moments requires very careful calibration as well as careful integration over the distribution function. Current electron data taken within interplanetary space are for the most part on a par with the ion data and as will be demonstrated in this paper, solar wind moments from the PEACE and CIS experiments are nearly interchangeable.

In interplanetary space the plasma is dominated by the solar wind. The solar wind consists of multiple electron populations which include the core, halo, superhalo, and the strahl (see Lin et al., 1997; Maksimovic et al., 2005, and references therein). In the vicinity of $1 \mathrm{AU}$, if the point of observation is also connected to the bow shock, the plasma may contain solar wind electrons which have been reflected off the bow shock (Wu, 1984) or post-shocked electrons which have leaked back into the upstream (Gosling et al., 1989). Because we will not be concerned with the exact source of these the latter two population of electrons in this paper, we will refer to any population that is moving back into the upstream as return electrons. Both the strahl and the return electron populations are highly field aligned, which allows them in most

Published by Copernicus Publications on behalf of the European Geosciences Union. 
cases to be easily distinguished and separated from the core and halo.

Indirect inference of plasma vorticity has been made in many studies, generally by coupling observations with specific models or simulations (e.g., see Goldstein et al., 2001). Deducing vorticity from a single-point measurement implies that only one scalar component can be estimated. A direct, volumetric determination can, in principle, deduce the full vector nature of the vorticity and also allow for dynamic changes in the vorticity to be followed with a time resolution commensurate with the time resolution of the moments. For example, Burlaga (1990); Burlaga and Richardson (2000); Burlaga et al. (2002); Veselovsky and Triskova (1990); Veselovsky (1990) have suggested the presence of a heliospheric vortex street in the interplanetary media using Voyager-2 data while the presence of vorticity on the flanks of the magnetosphere has been deduced from Cluster observations by several authors, usually when the IMF is northward (Hasegawa et al., 2004, 2006; Fairfield et al., 2007), but, more recently, also when southward Hwang et al. (2010).

In this paper we present direct measurements of vorticity in the electron plasma near $1 \mathrm{AU}$. These provide significant constraints on possible driving mechanisms and allow for reasonably unambiguous identification of the source. The measurements are made using the PEACE experiment on the four Cluster spacecraft. The paper is divided into two parts. In the first part we describe the methodology used to compute the plasma moments and the spatial derivatives. The vorticity is estimated directly from the spatial derivatives. In addition, we also show comparisons of the ion and electron moments as well as describe the method used to cross calibrate the electron moments across the four spacecraft. In the second part of the paper we present measurements of the electron vorticity over a two and a half hour period from 20 February 2003, when the spacecraft were upstream of the bow shock and for the most part within the foreshock. We will show that most of the observed vorticity can be explained by small deflections in the overall bulk velocity caused by rotations in the magnetic field. The deflections appear to be due to the strong coupling of the magnetic field with the strahl and return particle populations.

\section{Data}

This study uses data from five separate Cluster experiments. The electron data from PEACE forms the primary data set while data from the Fluxgate Magnetometer (FGM); Electric Field and Waves (EFW); Waves of High frequency and Sounder for Probing of Electron density by Relaxation (WHISPER); and CIS are used to verify the electron data, to describe local conditions, and to support conclusions. Below we provide a brief description of the PEACE experiment as well as how the data from the other experiments are used.
PEACE consists of two hemispherical electrostatic analyzers on each of the Cluster satellites (Johnstone et al., 1997). The two analyzers, designated HEEA (High Energy Electrostatic Analyzer) and LEEA (Low Energy Electrostatic Analyzer) are separated by $180^{\circ}$ in phase on the satellite and differ only in their geometric factors (HEEA has the larger geometric factor). Despite their acronyms, both can cover the energy range $0.6 \mathrm{eV}$ to $26 \mathrm{keV}$. We include data only from LEEA in this paper. The analyzers field of view are perpendicular to the spacecraft spin axis, i.e., approximately perpendicular to the GSE ecliptic. Each covers $180^{\circ}$ of polar angle in 12 sectors. The full $360^{\circ}$ of azimuth is covered in one rotation of the spacecraft allowing a three-dimensional snapshot of the electron distribution to be accumulated once per $\operatorname{spin}(\sim 4 \mathrm{~s})$.

Because of telemetry restrictions, PEACE generally returns only a subset of the total data collected on-board. Exactly what is returned depends on the instrument mode which can be separately commanded for both analyzers on each of the four spacecraft. The telemetry rate determines the frequency with which full three-dimensional distributions are downloaded. During the time intervals used in this paper, all satellites were operating in burst mode telemetry and PEACE was returning 3-D distributions every four seconds. The LEEA analyzers on all spacecraft were returning data in 3DXP1 mode (30 energy steps, 32 azimuth sectors, and 6 polar zones). The covered energy range was from 5 to $1050 \mathrm{eV}$.

Data from PEACE provide a full description of the local electron environment at each spacecraft. Moments formed from the data are used as input into an algorithm to compute a set of spatial parameters within the volume formed by the four spacecraft. This includes estimates of the electron compression and vorticity. EFW data provides a spin averaged spacecraft potential which is used to correct the energy bin limits of the PEACE energy steps prior to computing the electron moments. Both WHISPER and CIS data are used as a check the overall calibration of the PEACE analyzers. The PEACE-derived electron densities are compared against the WHISPER density determination made from the Langmuir plasma resonance while the bulk electron component velocities are compared against the CIS derived values for the ions. The latter comparisons can only be made on $\mathrm{C} 1$ and $\mathrm{C} 3$ due to failures of the CIS HIA experiments on $\mathrm{C} 2$ and $\mathrm{C} 4$. The status of the WHISPER experiment must also be monitored as when it is actively sounding it distorts the spacecraft potential and hence the moments. The magnetic field data is used to characterize the local plasma environment and in rotating moments into a magnetic field frame of reference. With the exception of the EFW potential data which was taken from data provided to the PEACE team, all data was obtained from the Cluster Active Archive (CAA). 


\section{Moment computations}

The moments associated with a velocity distribution function (VDF) are formed from numerical solutions of the equation:

$$
\langle M\rangle=\int_{0}^{2 \pi} \int_{0}^{\pi} \int_{0}^{\infty} f(v, \phi, \theta) v_{\mathrm{x}}^{l} v_{\mathrm{y}}^{n} v_{\mathrm{z}}^{m} v^{2} \sin \theta d v d \theta d \phi
$$

where $M$ is the moment (density, velocity, pressure, heat flux), $f$ is the plasma VDF in spherical coordinates, $v$ is the particle velocity, $\phi$ is the azimuthal angle, $\theta$ is the polar angle, $\left(v_{\mathrm{x}}, v_{\mathrm{y}}, v_{\mathrm{z}}\right)$ are the particle component velocities expressed in Cartesian coordinates and $(l, m, n)$ are exponents which range from 0 to 3 . Moments are generally expressed in Cartesian coordinates but evaluated in spherical coordinates and it should be understood that the Cartesian velocity components in Eq. (1) are to be converted to a spherical representation prior to integration. The exact values of the exponents in the equation are set by the moment being computed. Density is computed by setting all the exponents to 0 and the three components of the bulk velocity by setting either $l, n$ or $m$ to 1 and the remaining two exponents to 0 . Other combinations are used in the computation of the pressure and heat flux tensors neither of which is used in this paper.

The approach used to evaluate Eq. (1) depends to a large degree on how the data forming the VDF is taken. PEACE takes measurements within a discrete number of volumes in phase space, each of which has associated with it a unique energy, polar, and azimuth range. Together these bins form the $f(v, \phi, \theta)$ data matrix used in the moment integrals. Energy $(\varepsilon)$ is scanned sequentially from a preset maximum to minimum value during which time the spacecraft rotation is used to scan in azimuth $(\phi)$. The polar data $(\theta)$ are taken in parallel at each $(\varepsilon, \phi)$ measurement. Scans in energy are repeated throughout the spin. The number of energy sweeps in a spin, as well as the number of polar bins returned depends on the instrument mode. A complete $f(v, \phi, \theta)$ matrix is acquired once per spin. The measured matrix is contiguous in energy and polar angle but generally not in azimuth. This prevents a direct solution of Eq. (1) as a sum of integrals over the individual volumes without some assumptions.

The gap in azimuth arises from the time difference between successive measurements at the same energy step. This is illustrated in Table 1 which shows a pair of hypothetical energy sweeps each containing 5 bins. The energy bins are sampled in succession beginning at E1 and ending at E5 at which point the process repeats. During this time the spacecraft has rotated through an azimuth equivalent to $360\left(T_{\mathrm{s}} / T_{\mathrm{p}}\right)$ where $T_{\mathrm{s}}$ is the time between successive measurements of the same energy step (equivalent to the time required to complete an energy sweep) and $T_{\mathrm{p}}$ is the spacecraft spin period. The amount of rotation varies with mode but is generally between $5.6^{\circ}$ and $22.5^{\circ}$. The azimuthal width subtended by the energy measurement (assuming a $4 \mathrm{~s}$ spin) is $0.375^{\circ}$ plus the instrument azimuthal field of view $\left(2.8^{\circ}\right.$ for
Table 1. Origin of gaps in azimuth at a fixed energy in the VDF matrix.

\begin{tabular}{|c|c|c|c|c|c|c|c|}
\hline \multirow[t]{5}{*}{ E1 } & \multicolumn{2}{|c|}{ Azimuthal Gap } & E1 & & & & \\
\hline & \multirow[t]{4}{*}{ E2 } & \multicolumn{2}{|c|}{ Azimuthal Gap } & E2 & & & \\
\hline & & \multirow[t]{3}{*}{ E3 } & \multicolumn{2}{|c|}{ Azimuthal Gap } & E3 & & \\
\hline & & & \multirow[t]{2}{*}{$\mathrm{E} 4$} & \multicolumn{2}{|c|}{ Azimuthal Gap } & E4 & \\
\hline & & & & E5 & Azimut & Gap & E5 \\
\hline
\end{tabular}

LEEA and $5.3^{\circ}$ for HEEA). Only HEEA at the smallest gap provides a contiguous set of data in azimuth. The azimuthal gap in the data set used in this paper is $7.5^{\circ}$.

The standard procedure, when there is a gap in any direction, is to expand the measurement to fill the gap. This is the approach we will take with the gap in azimuth, however, rather than assume that the VDF measured within a given $(\varepsilon, \theta)$ is constant through the gap, as is often done, we assume that the VDF varies linearly in $\phi$ across the volume $i$ as:

$$
f_{i}(\phi)=A_{i} \phi+B_{i}
$$

Here $A_{i}$ and $B_{i}$ are given by:

$$
\begin{aligned}
A_{i} & =\frac{f_{i+1}(\phi)-f_{i}(\phi)}{d \phi_{i}} \\
B_{i} & =-A_{i} \phi_{i 0}+f_{i}(\phi)
\end{aligned}
$$

where $d \phi_{i}$ is the azimuthal width of the volume, $\phi_{i 0}$ is the azimuth at the leading edge of the volume, and $f_{i}$ and $f_{i+1}$ are the VDF values in the current and next volume elements.

With a contiguous set of data the preferred method of evaluation of Eq. (1) is to represent it as a sum of exact integrals over the individual measurement volumes. Substituting Eq. (2) into Eq. (1), the integral over a volume element $i$ becomes

$$
\left\langle M_{i}\right\rangle=\int_{\phi_{a i}}^{\phi_{b i}} \int_{\theta_{a i}}^{\theta_{b i}} \int_{V_{a i}}^{V_{b i}}\left(A_{i} \phi+B_{i}\right) v_{x}^{l} v_{y}^{m} v_{z}^{n} v^{2} \sin \theta d v d \theta d \phi
$$

This can be evaluated analytically for any $(l, m, n)$. The integral limits are those associated with the $i$-th volume.

One last step, prior to integration, is to correct the measured energies (and through them the velocities) for the spacecraft potential. The correction formula for the energy is:

$\varepsilon=\varepsilon_{M}-\Phi-1.5$

where $\varepsilon_{M}$ is the measured energy, $\Phi$ the spin averaged spacecraft potential obtained from EFW, and the 1.5 is the estimated contact potential between the probes and the ambient plasma (F. Mozer, private communication) in volts. Small differences in the actual contact potential from that used will not effect the conclusions drawn in this paper. 
The correction for potential presents two special cases which must be considered when solving Eq. (3); times when the volume element falls below the spacecraft potential and times when the spacecraft potential falls within the volume element. Handling the first case is straightforward; the volume integral is set to 0 and as such does not contribute to the overall integral. The second case, however, allows for several approaches. The easiest is to use the same approach as when the volume element is below the potential and set the volume integral to 0 . This is, however, not a very satisfying one. If the potential is near the upper edge of an energy bin then it can be argued that only a small fraction of the volume element would actually contribute to the integral and setting it to 0 would not be out of line. The same cannot be said, however, if the potential is closer to the lower edge of the energy bin where now a large fraction of the volume element would contribute to the integral. The approach we use to set the lower velocity limit in the integration to 0 and to replace its phase space density (which is not usable as it is contaminated to an unknown degree by the plasma below the spacecraft potential) with that measured in the next higher energy bin. We consider this to be a better solution than to totally ignore the volume in the overall integration.

The analytical solutions of Eq. (3) for the plasma density and bulk velocity along the three principal axes are given by:

$$
\begin{aligned}
N= & \frac{1}{3} \sum_{i=0}^{i=J}\left(v_{i b}^{3}-v_{i a}^{3}\right)\left(\cos \theta_{i a}-\cos \theta_{i b}\right) \times \\
& \left(\frac{A_{i}}{2}\left(\phi_{i b}^{2}-\phi_{i a}^{2}\right)+B_{i}\left(\phi_{i b}-\phi_{i a}\right)\right) \\
V_{\mathrm{x}}= & \frac{1}{4 N} \sum_{i=0}^{i=J}\left(v_{i b}^{4}-v_{i a}^{4}\right)\left(\frac{1}{2}\left(\theta_{i b}-\theta_{i a}\right)-\frac{1}{4}\left(\sin \left(2 \theta_{i b}\right)-\right.\right. \\
& \left.\left.\sin \left(2 \theta_{i a}\right)\right)\right) \times\left(A _ { i } \left(\cos \phi_{i b}+\phi_{i b} \sin \phi_{i b}-\cos \phi_{i a}\right.\right. \\
& \left.\left.-\phi_{i a} \sin \phi_{i a}\right)+B_{i}\left(\sin \phi_{i b}-\sin \phi_{i a}\right)\right) \\
V_{\mathrm{y}}= & \frac{1}{4 N} \sum_{i=0}^{i=J}\left(v_{i b}^{4}-v_{i a}^{4}\right)\left(\frac{1}{2}\left(\theta_{i b}-\theta_{i a}\right)-\frac{1}{4}\left(\sin \left(2 \theta_{i b}\right)-\right.\right. \\
& \left.\left.\sin \left(2 \theta_{i a}\right)\right)\right) \times\left(A _ { i } \left(\sin \phi_{i b}-\phi_{i b} \cos \phi_{i b}-\sin \phi_{i a}\right.\right. \\
& \left.\left.+\phi_{i a} \cos \phi_{i a}\right)-B_{i}\left(\cos \phi_{i b}-\cos \phi_{i a}\right)\right) \\
V_{\mathrm{z}}= & \frac{1}{4 N} \sum_{i=0}^{i=J}\left(v_{i b}^{4}-v_{i a}^{4}\right)\left(\frac { 1 } { 2 } \left(\sin ^{2} \theta_{i b}-\right.\right. \\
& \left.\left.\sin \theta_{i a}^{2}\right)\right)\left(\frac{A_{i}}{2}\left(\phi_{i b}^{2}-\phi_{i a}^{2}\right)+B_{i}\left(\phi_{i b}-\phi_{i a}\right)\right)
\end{aligned}
$$

where $J$ in the summation is the total number of volume elements which depends on the instrument mode.

The PEACE moments are computed in the instrument reference frame. These are then nominally rotated into the GSE frame of reference and if required rotated again into a magnetic field frame of reference.

An important consideration when working with Cluster plasma data is the status of the WHISPER experiment. When WHISPER is actively sounding it tends to distort the spacecraft potential making any moments derived during this time period unreliable. For this reason, moments computed during spins when WHISPER is sounding are flagged and dropped from all subsequent analysis. In general the gaps these leave in the time series are filled by a linear interpolation using the moments to either side.

Viñas and Gurgiolo (2009) have compared the above method of moment evaluation with one in which the moments are evaluated using a spherical harmonic fit to the VDF. The two methods give nearly identical results through the computation of the pressure tensor. Heat flux was not evaluated. The comparison shows that for the most part further interpolation of the velocity and polar angle through the individual bins yields negligible returns.

\section{Computing spatial derivatives}

Determination of both the plasma compression $(\nabla \cdot \mathbf{V})$ and vorticity $(\nabla \times \mathbf{V})$ involves the computation of spatial derivatives. Spatial derivatives of a quantity $Q$ can be computed provided that it is known at a minimum of 4 non-coplanar spatial locations, a condition met by the four Cluster spacecraft. With only four data points we are limited to assuming a linear solution to the variation of the quantity across the volume defined by the spacecraft as:

$Q_{i}=a_{i}+b_{i} X+c_{i} Y+d_{i} Z$

where $Q$ is the quantity being fit, $i$ is used to represent component (x, y or $\mathrm{z}),(a, b, c, d)$ are a set of unknown coefficients, and $(X, Y, Z)$ are the spacecraft position coordinates. For any $Q$ there are 4 equations of the form (8), one per spacecraft.

To give an explicit example consider the solution for $V_{\mathrm{x}}$. (Solutions for $\mathrm{Vy}$ and $\mathrm{Vz}$ are similar.) This is given by the coupled set of equations:

$$
\begin{aligned}
& C 1: V_{x 1}=a_{x}+b_{x} X_{1}+c_{x} Y_{1}+d_{x} Z_{1} \\
& C 2: V_{x 2}=a_{x}+b_{x} X_{2}+c_{x} Y_{2}+d_{x} Z_{2} \\
& C 3: V_{x 3}=a_{x}+b_{x} X_{3}+c_{x} Y_{3}+d_{x} Z_{3} \\
& C 4: V_{x 4}=a_{x}+b_{x} X_{4}+c_{x} Y_{4}+d_{x} Z_{4}
\end{aligned}
$$

which can be solved by any number of common analysis techniques.

Using solutions for the coefficients to the component velocity equations the compression and vorticity become:

$$
\begin{aligned}
& \nabla \cdot \mathbf{V}=b_{x}+c_{y}+d_{z} \\
& \Omega=\nabla \times \mathbf{V}=\left(c_{z}-d_{y}\right) \widehat{x}+\left(d_{x}-b_{z}\right) \widehat{y}+\left(b_{y}-c_{x}\right) \widehat{z}
\end{aligned}
$$


With only a linear fit to the data possible, both the divergence and curl are independent of position.

Possible errors in the computation of the spatial derivatives can be traced to five main sources: problems with timing, errors in the position variables, errors in the moment variables, inter-spacecraft calibration, and how well the data can be represented by a linear approximation. A detailed discussion of many of the above sources can be found in Chanteur (1998); Vogt and Paschmann (1998). How we handle such errors specific to this analysis are described below.

Timing errors arise due to the fact that the four Cluster spacecraft are not spin synchronized; that is similar measurements on each spacecraft do not start and end at the same time. Small differences in spin rate between the four spacecraft add to this. To compensate for this we place all of the moment data into a set of common time grids prior to usage. A time grid is a continuous set of cells all with a common width $\delta \mathrm{t}$. Each measurement has its own time grid and corresponding normalization grid and all time grids have identical timing definitions. For the purposes of this study the grid cell widths were chosen to be $4 \mathrm{~s}$, approximately the average spacecraft spin period. In general each computed moment will overlap two cells within the its grid unless the cell start and stop times happen to exactly match the measurement start and stop times. Cells will contain the time weighted data of the measurements which overlap it. The cell values are renormalized at the end of the data acquisition and prior to any usage. As a specific example consider a cell in the time grid that runs from $T$ to $T+4$ and a measurement $F$ that runs from $T+1$ to $T+5$. The cell contains $75 \%$ of the measurement (with $25 \%$ being contained in the next cell). The cell value is then incremented by $0.75 F$ and the corresponding normalization by 0.75 . The final renormalized value assigned to the cell is its value divided by its corresponding normalization value. This scheme allows for arbitrary numbers of measurements to be contained within a single time cell. An excellent overview of the methods and errors associated with aligning non-synchronized data sets can be found in Harvey and Schwartz (1998).

Errors due in the spacecraft positions are generally but not always negligible. Cluster positional errors are on the order of $1 \mathrm{~km}$ at apogee and decrease from this through perigee (Volpp and Sieg, 2010). The error is important when the spacecraft component separations are small. While the computation of the spatial derivative is made using the component spacecraft separations which can at times be quite small, even 0 if two spacecraft lie in the same plane, the total error is a combination of the errors between all spacecraft. This is generally on the order of $2 \%$ when the spacecraft in close formation (on the order of $100 \mathrm{~km}$ average separation) and decreased to under $0.2 \%$ when the spacecraft have separations on the order of $1000 \mathrm{~km}$.

Errors in the measurements themselves are difficult to estimate as they arise from a number of sources. Some of the more important include: statistical fluctuations in the mea- sured countrates; errors in the calibration coefficients; and aliasing within the full 3-D eVDF resulting from changes which occur on a time frame faster than the acquisition time. These and more have been covered in depth by multiple authors, for example see Paschmann et al. (1998).

To estimate errors in velocity moments due to statistical variations in the countrates we selected a typical eVDF, randomized the measured countrates about their mean assuming a Gaussian frequency distribution, and then computed the moments. This process was repeated 2000 times using the same base eVDF. Analysis of the spread in the resultant velocity moments gave a mean error of close to $6 \%$ (half width divided by the mean).

Estimation of the errors due to problems in the calibration can be obtained by comparisons of the moments themselves with comparable moments derived from other experiments, generally WHISPER for electron density and in the solar wind, CIS for the velocity moments. This is taken up in more detail in Sect. 5 with the results indicating that the errors of this nature are probably small.

Time aliasing in the moments which occurs when there are large variations in the VDF on time scales faster or commensurate with the spin period can pose a significant source of error. These types of variations are generally associated with rapid boundary crossings and regions of high frequency turbulence. With solar wind electrons rapid rotations in the magnetic field can also lead to time aliasing as this is reflected in changes in the flow directions of the strahl and return populations. Errors due to time aliasing do not lend themselves to easy estimation. They occur only at select times in the data and their associated error is a function of what effect they have of the measured eVDF.

Cross-calibration of the instruments across the four spacecraft can introduce significant errors in the spatial derivatives if not taken into account and corrected. The approach we have taken to cross-calibrate the moments across the spacecraft is discussed in Sect. 6.

Probably the most important error associated with the computation of the spatial derivatives is contained in the assumption established in Eq. (8); that the data is linearly varying across the volume defined by the spacecraft. This is probably never the case and the question really to be asked is how close to linear is the variation? Unfortunately this is not an answerable question.

The use of a linear fit should be a good approximation at small spacecraft separations and anytime the spacecraft separations are smaller than the basic spatial scale lengths of interest in the data. However, when the average spacecraft separation is much larger than the spatial scale lengths of interest significant errors can arise. For example, consider the case where there is a sharp, narrow jump in the velocity within the volume defined by the spacecraft with no spacecraft in the transition region. Each spacecraft sees a velocity of either $V_{1}$ or $V_{2}$ depending upon which side of the transition it is located. The linear fit simply spreads the localized gradient 


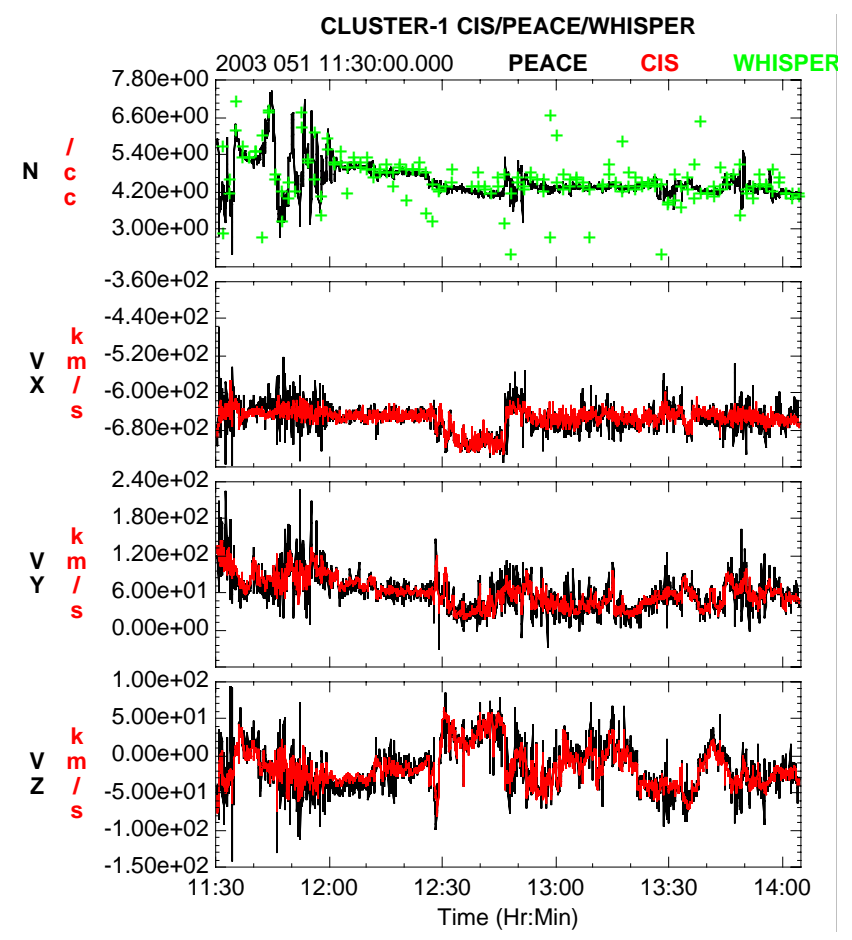

Fig. 1. Comparison of PEACE (black), CIS (red) and WHISPER (green) derived plasma moments in the solar wind from 20 February 2003 from 11:30:00 to 14:05:00 UT. Data was obtained from the C1 satellite.

out over the entire volume which produces an underestimation in all of the spatial derivatives. This underestimation increases with increased spacecraft separation and decreases as the spacecraft separation approaches a distance comparable with the scale length of the transition region.

To compound the problem, if the spacecraft configuration forms a highly irregular tetrahedron, the spacecraft separations along the GSE axes (the axis along which the vector moments are computed) can be quite diverse. (Even with the spacecraft in a perfect tetrahedral configuration the scale lengths along the principle axes are generally unequal.) This allows for cases where the linear fit may actually be a good representation along a subset of the three axes, but not all three. It also means that when there is underestimation of spatial derivatives, the amount of underestimation will probably be different along each axis. This difference will vary with the quality of the tetrahedron formed by the spacecraft and hence with orbital position.

The inclination then might be to restrict analysis to those cases where the spacecraft are closely separated to attempt to always be within the spatial scale lengths of the data. This, however, brings with it its own set of errors. The closer the spacecraft are to one another the less variation there should exist in similar measurements. (As two spacecraft approach each other common measurements should converge to a single value.) Errors in instrument calibration and cross calibra- tion and statistical errors begin to assume a dominant role in the accuracy of the spatial derivative determination. This is generally preferable to the case where the spacecraft separation is larger than the spatial scale lengths unless the gradients are extremely small.

To conclude, while a detailed estimate of the total error associated with the computation of the divergence and cross product of the electron velocity is not practical, a standard propagation of error analysis of just the statistical errors associated with the measured parameters which enter into the computations suggests that a base $15 \%$ error in the divergence and a base $12 \%$ error in the cross produce would not be out of line. Errors from other sources discussed above would increase these when present.

\section{Data quality}

The use of electron moments to represent the solar wind raises concerns about accuracy. Much of these, if not all, have a heritage from dealing with older instruments and data sets. Concerns generally center on the energy range of the solar wind electrons which may extend below the instrument energy range, from contamination by spacecraft photoelectrons, from the influence of spacecraft charging, and, perhaps most importantly for solar wind observations, the difficulty in pulling the moments out of a thermal background that is one to two orders of magnitude greater than the velocity moments. All these situations can introduce significant errors into the computed electrons moments. These problems are generally not present in ion data and thus moment construction from ion data is much more straightforward and significantly less likely to have large errors. With the loss, however, of the $\mathrm{C} 2$ and C4 CIS HIA heads this cannot be done for computing spatial derivatives. Most new electron instruments, such as PEACE extend to low enough energies (especially given the boost in energy by the spacecraft potential) and are sufficiently well calibrated that such concerns should no longer be a problem. That PEACE is able to provide moments commensurate with the ion measurements is shown in Fig. 1.

Figure 1 is a comparison of plasma moments obtained from PEACE (black traces) with those obtained from WHISPER (green + ) and CIS (red traces) over the time interval to be looked at. The top panel shows the derived electron density and the following three panels, the components of the bulk velocity. All data is from $\mathrm{C} 1$. The figure shows that, overall, the electron and ion derived velocities are on a par with one another and that the PEACE measured density matches that derived from WHISPER. It should be noted, however, that there is somewhat more noise in the electron data that probably originates in the subsonic nature of the electron fluid, as noted above, however, some may be the result of time aliasing (changes in the distribution between the beginning and ending of the full 3-D measurement). 


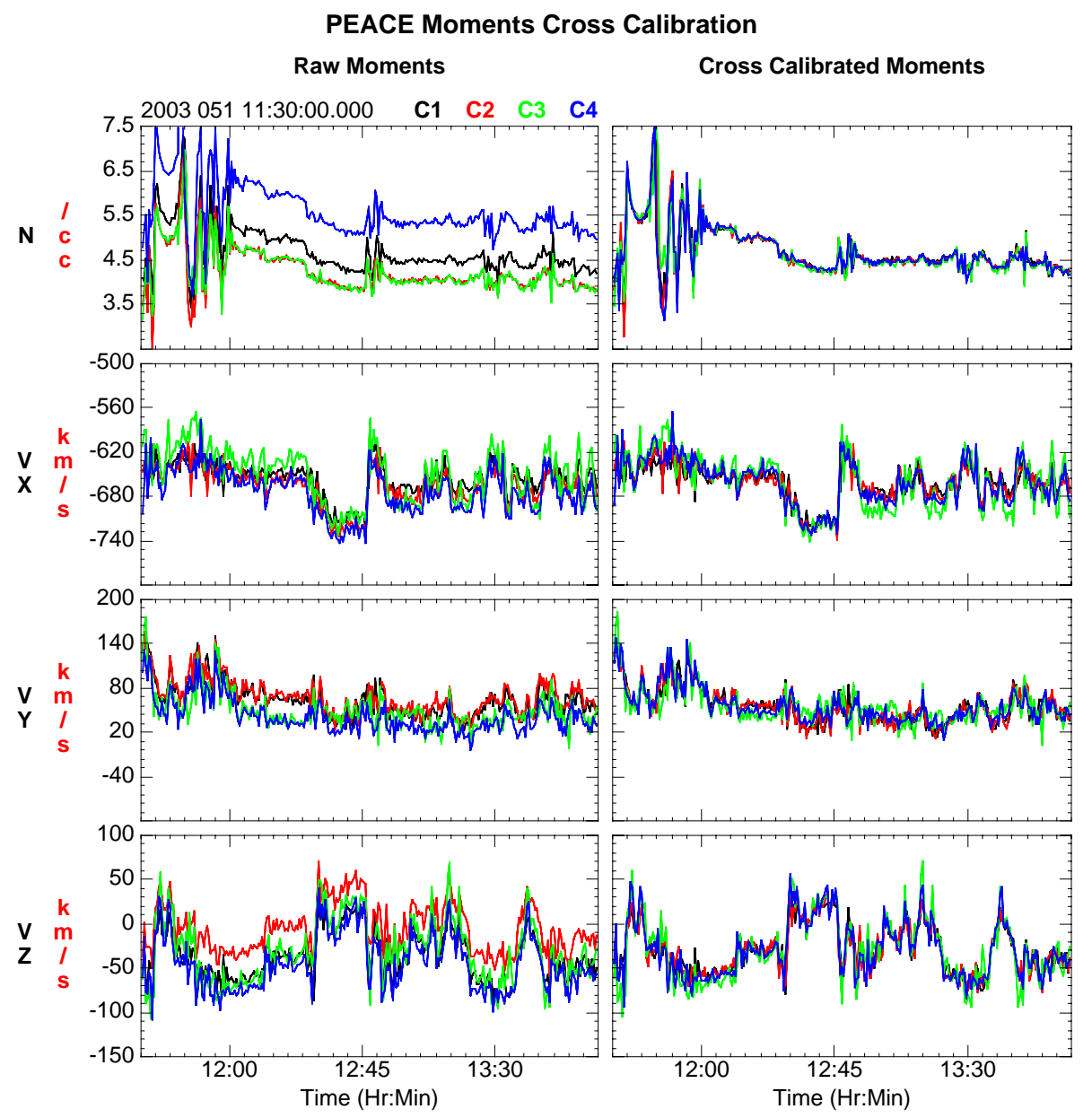

Fig. 2. Cross calibration of PEACE moments across the spacecraft.

\section{Cross calibration}

Computations of spatial derivatives require not only that individual instruments be well calibrated but also that they be calibrated across the various spacecraft. Minimal variations in the instrument calibrations result in DC offsets of the individual moments between the spacecraft. These differences introduce gradients across the measurement volume which in turn translate to DC offsets in the computed spatial derivatives. These can be significant when the spacecraft separation is small.

The purpose of cross calibration is to reduce the differences in calibration which may exist and to therefore minimize any existing DC offsets in the data between various spacecraft. Cross calibration of PEACE across the spacecraft is done on the moment level. If the instruments are all individually well calibrated the moments between spacecraft in the solar wind should closely resemble one another. To remove any DC offsets, the average of each moment is computed over a time period long enough to ensure that the averages are not influenced by short term variations in the data. For this study the average was taken over the entire event shown in Fig. 1, about $2.5 \mathrm{~h}$. In general the time period should be at least an hour in duration. The average moments from either $\mathrm{C} 1$ or $\mathrm{C} 3$ (whichever shows the closest match to the WHISPER electron density and CIS velocity) are used as anchors allowing DC or multiplicative shifts to be computed which are then used to shift the moments to the anchor moments. In general densities are multiplicatively shifted and velocities DC shifted. Figure 2 shows the density and velocity components for the 20 February 2003 event. Each panel shows corresponding moments from all four spacecraft. The moments in the left-hand column are those computed prior to cross calibration and those in the right-hand column of plots are the same moments after cross calibration.

The scatter in the density plots from the four spacecraft is the result of differences in the assumed individual analyzer gains which with the exception of $\mathrm{C} 1$ are not well set for this time period. The instrument gain however affects only the density computation and acts to multiplicatively shift the values either up or down by a constant factor. It has no affect on the velocity computations which are the crux of this paper. 


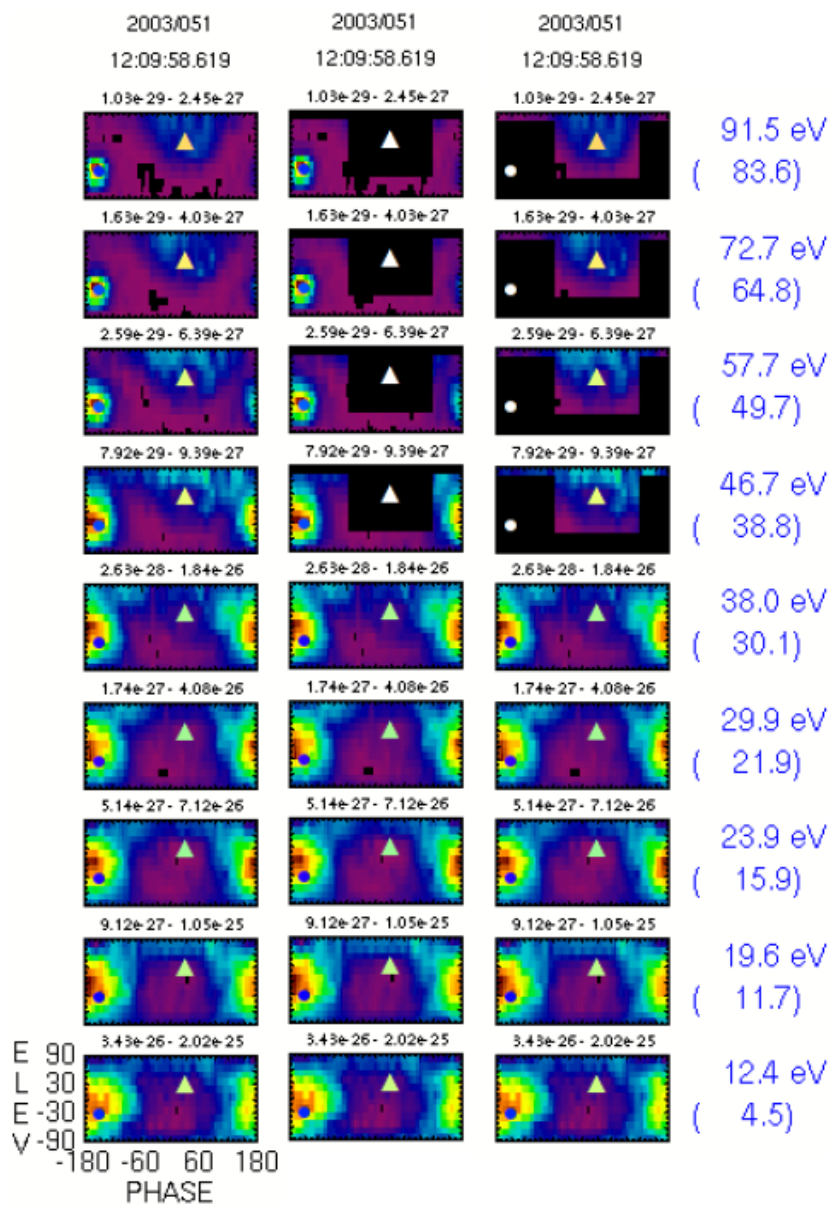

Fig. 3. A set of phi/theta plots illustrating how various volumes in phase space $\left(\mathrm{s}^{3} \mathrm{~cm}^{-6}\right)$ can be defined to isolate specific populations of particles.

The removal of DC shifts in the moments is seen in the second column of plots in the figure. This greatly tightens the correlations between the moments. As would be expected if the differences in density are gain based, the density curves show excellent correlation after cross calibration.

The basis for the DC shifts in the velocity are not clear. Presumably these arise from the how the MCP efficiencies are set on the various spacecraft. The MCP efficiencies exist per energy channel for each analyzer which makes any guess as to the source of the DC offsets beyond the scope of this paper. A DC rather than a multiplicative offset was used simple because it was better at cross calibrating the data.

\section{Separating populations}

The electron solar wind consists of multiple populations: the core, halo, superhalo (well above the PEACE energy range), and strahl. When the measurement point is connected to the bow shock there may also be a return population present in the VDF. At times it is expedient to be able to separate these populations and to compute their moments individually. The method used to separate the populations needs to be reasonably fast, straightforward, and able to be automated so that it can be carried over large time intervals out without intervention.

Our preferred method is to define volumes of interest in velocity space and then to integrate over the limits of the volumes. The volumes themselves are dynamically established and can to a high degree be used to isolate most of the populations involved. How this is done is illustrated in Fig. 3.

Each plot in the figure is a map in $(\phi, \theta)$ space of the eVDF measured at a fixed energy. This is shown in a PEACE based coordinate system in which $\theta$ is the angle of the LEEA aperture with the spacecraft spin axes $\left(\theta=0^{\circ}\right.$ is normal to the spin axis) and the plane formed by $\phi=0$ and the spin axis contains the sun. The Cluster spin axis is nearly parallel to the negative GSE Z-axis.

The particle velocities in the plots are shown in the plasma flow frame (which plots the measured intensity against the complement of the normal analyzer aperture angles). In this frame the core/halo is observed at a phase angle of $\pm 180^{\circ}$ which is anti-sunward flow rather than $0^{\circ}$ when the analyzer aperture is pointing at the sun. The center energy corresponding to each row of plots is shown at the right where the values in parentheses are the potential corrected energies. Because the color range is individually autoscaled for each plot you cannot simply compare intensities between plots by color. The minimum and maximum scaling is given above each plot. The solid triangle and circle in each plot are projections of the head and tail of the spin averaged magnetic field, respectively.

A column of plots represents a single VDF taken during a single spin. In this case each column of plots shows data from the same spin for the purpose of illustrating the use of different maskings to form an integration volume. The energies plotted within a column in the figure are a subset of the entire set of energies returned. With the exception of the lowest energy step shown, the energies are consecutive.

The first column of plots shows the data without any masking. The core-halo population is seen from $38.0 \mathrm{eV}$ down, moving anti-sunward and centered on $\phi= \pm 180^{\circ}$. Sitting above the core-halo in the plots is the strahl. Notice that at about $46.7 \mathrm{eV}$ the center of the distribution shifts from the sun centered line to the magnetic field and by $56.7 \mathrm{eV}$ the distribution is fully field-aligned. The transition occurs as the strahl begins to dominate over the halo. Also beginning at about $46.7 \mathrm{eV}$ there is a weak sunward moving field-aligned population centered on the triangle. This is a return electron population and indicates that the local field has a connection to the bow shock.

The second column of plots shows the application of a mask to remove the return electrons. The mask is centered and anchored on the head of the magnetic field and all VDF values within the masked region are set to 0 . Anchoring masks to the magnetic field allows them to follow the field 


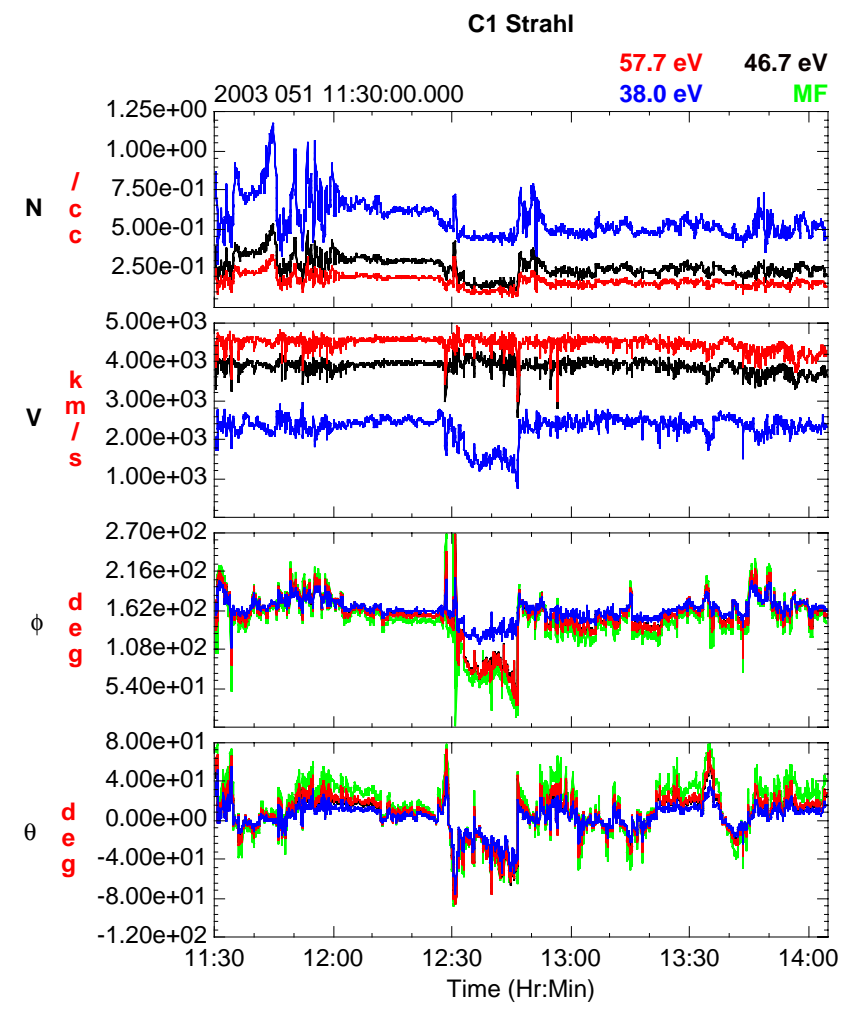

Fig. 4. Plots of the electron density and spherical velocity components for integrations starting a $38.0 \mathrm{eV}$ (blue), $46.7 \mathrm{eV}$ (black) and $57.5 \mathrm{eV}$ (red). Also shown are the angular components of the magnetic field (green).

and stay centered on the field aligned populations. Integrating over the masked VDF gives the full solar wind moments less the return population. By separating the integration into two, the first from 0 to $38.0 \mathrm{eV}$ and the second from $46.7 \mathrm{eV}$ to $\infty$, we can further separate the core/halo from the strahl. (Understand 38.0 to indicate the upper edge of that energy channel and 46.7 to indicate the lower edge of that energy channel so that the integrals are contiguous.)

The third column of plots shows masking of the strahl similar to the masking of the return particles. Integrating from $46.7 \mathrm{eV}$ to $\infty$ gives the moments associated with the return population.

For the time period being looked at in this paper we consider the strahl to run from $46.7 \mathrm{eV}$ up in energy and the core/halo from $38.0 \mathrm{eV}$ and down. It is obvious that at the break energy there is still some halo signature, however as seen in Fig. 4 this appears to be minimal.

Figure 4 shows the strahl moments computed from 38.0, 46.7 , and $57.7 \mathrm{eV}$ to infinity. The upper panel in the figure is the density followed by the spherical components of the velocity. There are two features to look for in the plots that would suggest a transition from a nearly pure strahl VDF to one that includes a significant amount of halo electrons; the deviation from a purely field aligned flow and a

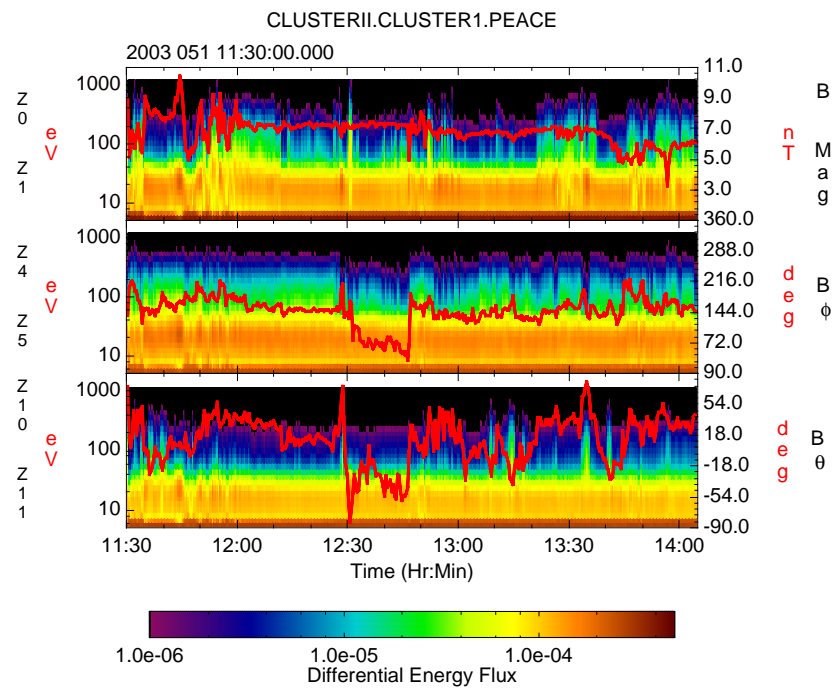

Fig. 5. Spectrograms of differential energy flux ( $\mathrm{ergs}^{-2} \mathrm{~cm}^{-\mathrm{s}-\mathrm{str}-}$ $\mathrm{eV}$ ) from three heads of the PEACE LEEA instrument on $\mathrm{C} 1 \mathrm{cov}-$ ering the time period of the event being analyzed. The upper and lower panels are from the extreme upward and downward pointing heads while the center panel is from a near equator looking head. The red traces are overlays of the spherical GSE components of the magnetic field.

large jump in density. Both of these occur with the inclusion of the $38.0 \mathrm{eV}$ electrons. For the most part the field is nominally radial, however, there is a large rotation between 12:30 and 12:50 UT and it is exactly in this interval where one can see the velocity moves significantly away from being field-aligned when the $38.0 \mathrm{eV}$ particles are added to the integral. This is seen primarily in the $\phi$ component. With only six returned polar bins $\left(30^{\circ}\right.$ bin widths as opposed to the $11.25^{\circ}$ azimuthal bin widths) at this time the resolution is insufficient to show the separation in $\theta$ other than to suggest that the polar flow angle does move toward the ecliptic plane. The large jump in density when including the $38.0 \mathrm{eV}$ electrons is another indication of the inclusion in the VDF of a large halo population.

\section{Observations}

Figure 5 shows spectrograms from three of the PEACE LEEA polar bins on Cluster-1 covering the interval 11:30 to 14:04 UT on 20 February 2003 (day 51). Magnetic field data in spherical coordinates is overlaid in red. The upper and lower spectrograms contain data from the extreme polar bins (both looking near perpendicular to the ecliptic plane) while the center panel contains one of the two ecliptic band polar sectors. The spacecraft separation for the same time period is shown in Fig. 6. Total distance between the spacecraft varies from 3000 to $5700 \mathrm{~km}$ while the configuration geometric quality factory, $Q_{\mathrm{GM}}$, as defined in Robert et al. 


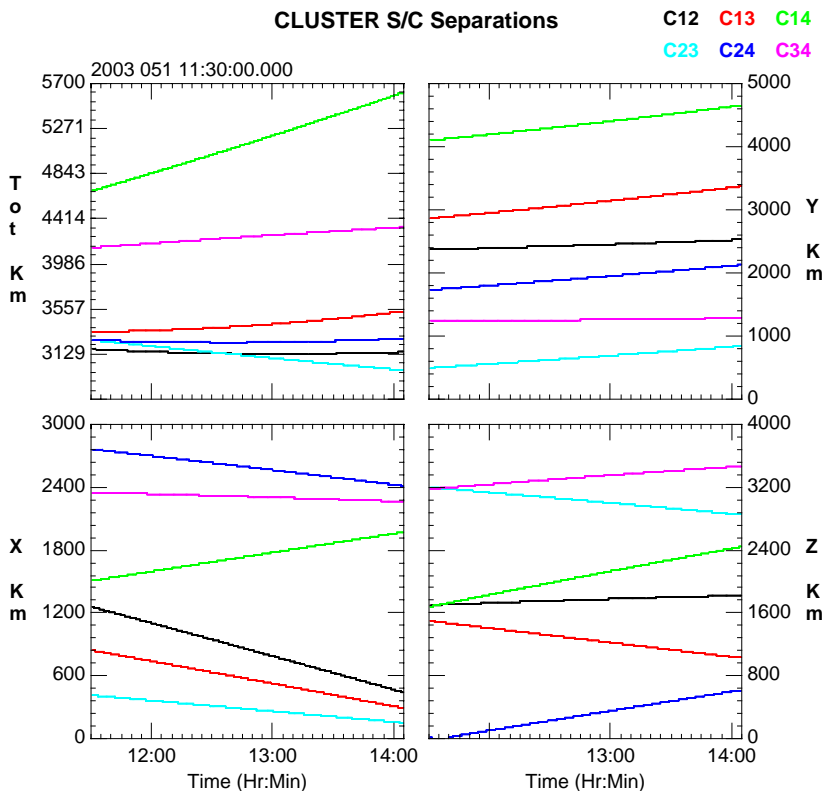

Fig. 6. Total and GSE component spacecraft separations over the time interval being analyzed.

(1998), varies from 2.79 to 2.43 . The latter indicates that the spacecraft are in a reasonable tetrahedral configuration.

There are several features to note in Fig. 5. In the center panel the high energy particles above $50 \mathrm{eV}$ are a combination of return electrons moving sunward and the strahl which are moving anti-sunward (refer back to the first column in Fig. 3). The former population can be used as an indicator of when the spacecraft is in the foreshock although this is not foolproof since you can be connected with no return signal. There is a large rotation in the field between 12:30 and 12:50 UT which is clearly seen in $B_{\phi}$ and several sharp rotations in $B_{\theta}$, notably just before 12:30 UT and at 13:35 UT. These rotations in $\theta$ move both the return and strahl signatures (which are field aligned) out of the ecliptic and to more extreme polar angles. This is seen in Fig. 5 as sharp decreases in the $>50 \mathrm{eV}$ particles in the middle panel coupled with increases in the high energy signature in the upper and lower panels. Because the strahl and reflected particles are counter-streaming they shift in opposite directions; the strahl shifts to the upper panel while the reflected particles move to the lower panel.

Plots of the different spatial derivatives for the 20 February event are shown in Fig. 7. The upper panel contains the reduced $\mathrm{C} 1$ parallel velocities of the return (black) and strahl (red) populations and allows easy identification of periods when both populations are present versus when only the strahl is present. The reduced velocity is the measured velocity multiplied by the ratio of its density to the total electron density. This is effectively the contribution of the population to the total parallel velocity. Note that the return velocity drops to a non-zero baseline when the population is absent.
Cluster Plasma Volumetric Parameters

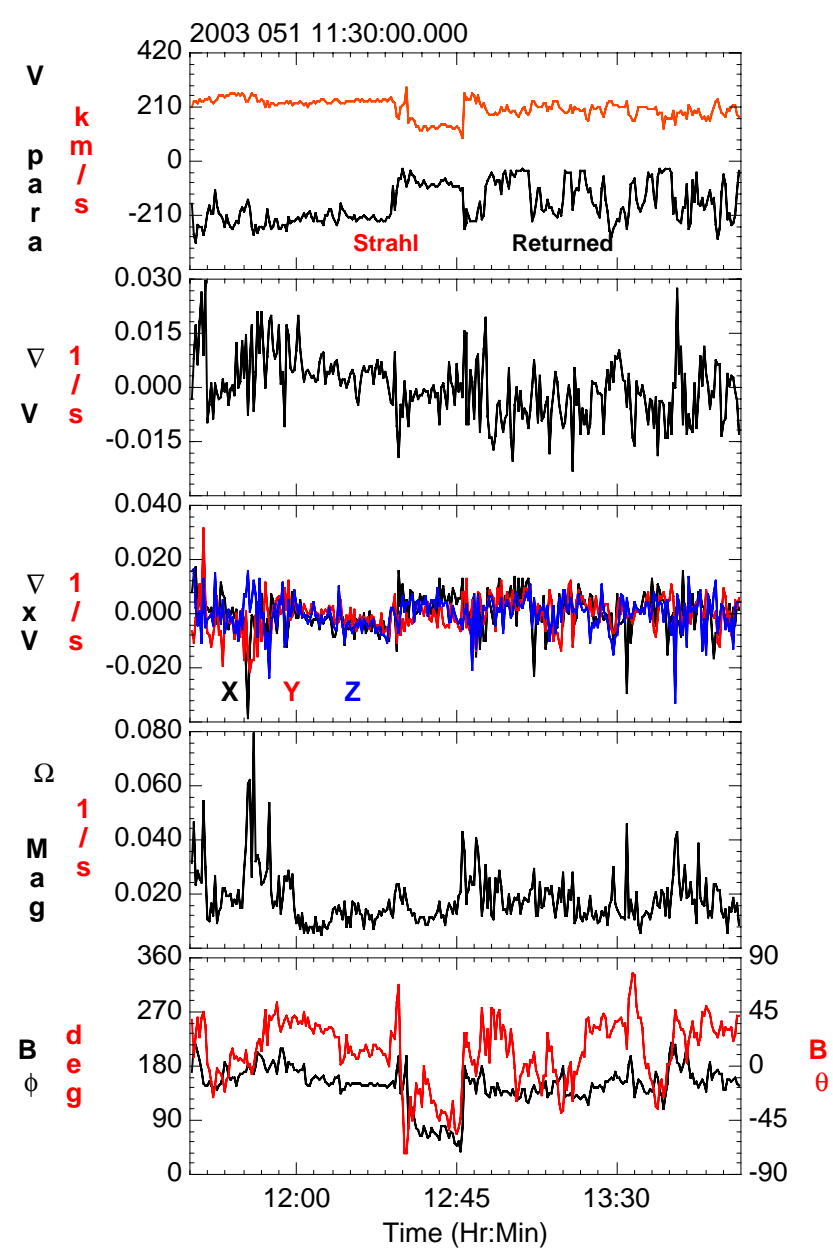

Fig. 7. In descending order: the reduced parallel velocity of the C1 strahl and return electrons, the electron compression, three GSE components of the electron vorticity, the magnitude of the vorticity and the $\phi$ and $\theta$ GSE components of the $\mathrm{C} 1$ magnetic field.

This is due to the presence of low intensity noise within the mask region. The succeeding panels in the figure show the electron compression, the components of the vorticity, the magnitude of the vorticity, and the phi and theta angles of the $\mathrm{C} 1$ magnetic field. All plots of vorticity together with the plot of the compression in this figure and successive figures are for the total electron population. High time resolution plots of the magnetic field data for all spacecraft (not shown) indicate that the spatial scale associated with rotations in the magnetic field are much larger than the spacecraft separations.

While the divergence of the velocity is not explicitly considered in this paper it is interesting to note that the electron population appears to be expansive in the presence of return electrons and compressive when they are absent. It is not entirely obvious why this should occur. 


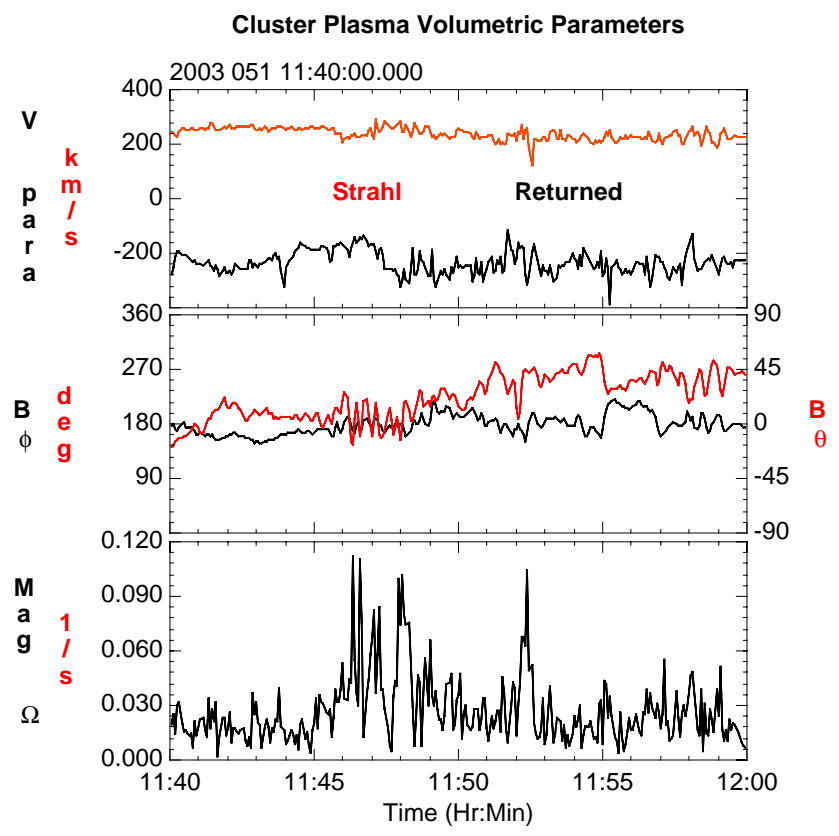

Fig. 8. High resolution plot of the parallel strahl and return velocities, the $\phi$ and $\theta$ GSE components of the C1 magnetic field and the magnitude of the vorticity for the time surrounding the initial large increase in vorticity.

Multiple instances of enhanced vorticity are readily seen in the plot of the vorticity magnitude the most intense of these occurring between 11:40 and 12:00 UT. The following three plots show higher resolution plots of specific time periods within the interval to better show what relationships exist between increases in vorticity and the local conditions. The times were selected to show the vorticity within very different upstream conditions.

Figure 8 covers the time period of the first set of enhancements in the vorticity. There are return particles present during the entire time period. The vorticity seen between 11:46 and 11:49 occurs in the presence of a set of magnetic waves as seen in $B_{\theta}$. The large increase in vorticity near 11:52 also occurs in conjunction with a large rotation in $B_{\theta}$. Smaller increases in the vorticity also appear to be correlated with rotations in the magnetic field.

Figure 9 covers the time of the major field rotation between 12:30 and 12:46 UT. During this time period there is no evidence of return particles at the spacecraft. While there is evidence of enhanced vorticity at the edges of the rotation it is by no means as intense as that seen in Fig. 8 or even that seen later in this plot. As in the previous plot many of the increases in vorticity appear to be well correlated with field rotations.

The next figure shows a time period where the spacecraft are moving in and out regions containing return particles. If there is a correlation of vorticity with the boundaries separating regions which contain return electrons and those that

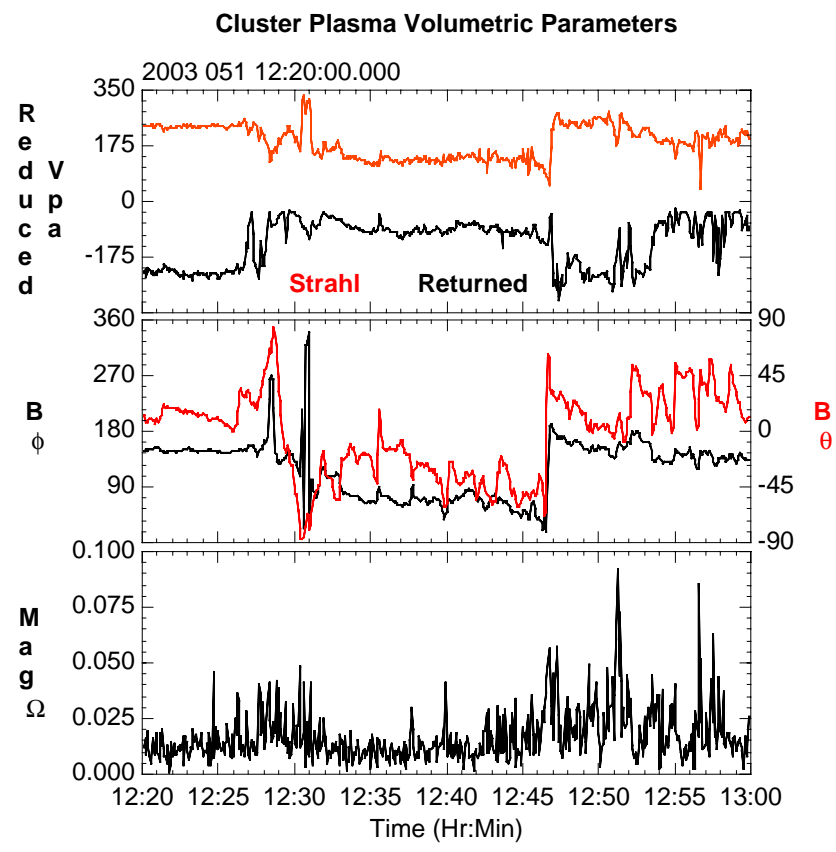

Fig. 9. High resolution plot of the parallel strahl and return velocities, the $\phi$ and $\theta$ GSE components of the C1 magnetic field and the magnitude of the vorticity for time around the large magnetic field rotation.

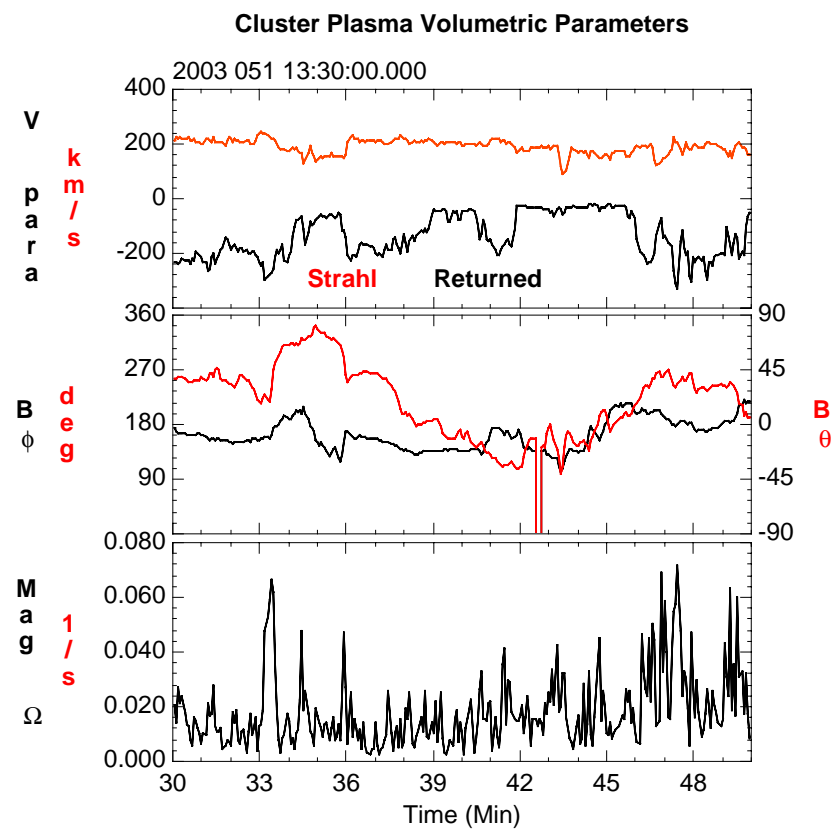

Fig. 10. High resolution plot of the parallel strahl and return velocities, the $\phi$ and $\theta$ GSE components of the $\mathrm{C} 1$ magnetic field and the magnitude of the vorticity for the time period when the return electrons are waving across the spacecraft.

don't it is hard to determine as most boundaries occur as the result of field rotations sweeping the boundaries across 


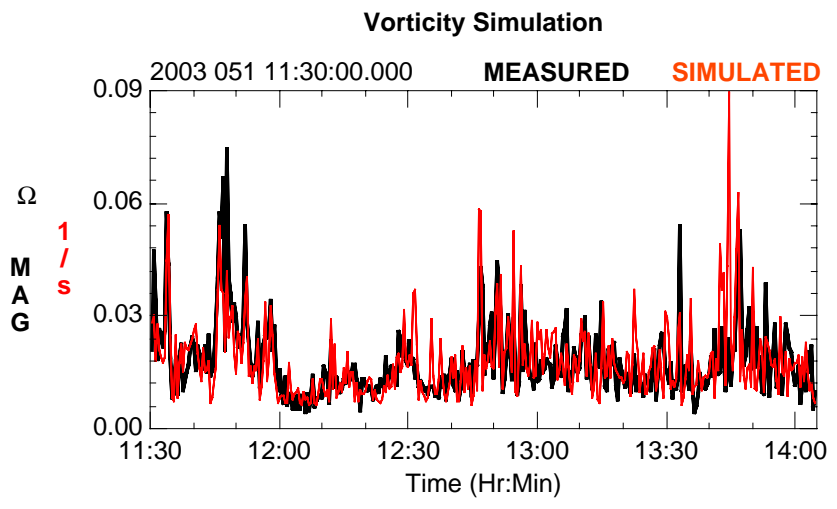

Fig. 11. Measured electron vorticity (black) compared with the vorticity which would be produced by changes in a constant field aligned velocity across the four spacecraft.

the spacecraft. One exception may be the enhancement at 13:34:30 UT, which does not appear to be correlated with any major field rotation but occurs in conjunction with a small reappearance of return particles. The increase in vorticity near 13:43 is one of the few seen during this event which occurs in the absence of return particles.

\section{Discussion}

The strong correlation between the electron vorticity and rotations in the magnetic field suggests a connection to either the strahl and/or the return populations. Both populations are highly field-aligned however it should be noted that the return populations often show non-gyrotropic features as can be seen in Fig. 3 which results in some perpendicular flow in their velocity moments. Directional changes in the magnetic field will translate to directional changes in the flow velocities of both components. Should these changes result in deflections in the total fluid velocity then a vorticity will exist provided that the magnetic field variations are local and propagate across the spacecraft (i.e., there are different deflections at each satellite).

The role of the rotations in the magnetic field can be seen in Fig. 11. In the figure the red trace is the result of a simple simulation in which there is assumed to exist a constant $200 \mathrm{~km} \mathrm{~s}^{-1}$ field aligned velocity at each of the four spacecraft. The GSE component velocities at each spacecraft are then computed by decomposing this velocity using the measured field orientations. The vorticity is then computed using the actual spacecraft separations.

The overall qualitative agreement between the simulated and measured vorticity demonstrates how strongly coupled the existence of vorticity is to rotations in the magnetic field. The good quantitative agreement is surprising. The constant $200 \mathrm{~km} \mathrm{~s}^{-1}$ field aligned velocity used in the simulation is simply an average velocity of the strahl over the time period. This does not mean that the strahl or for that matter the return

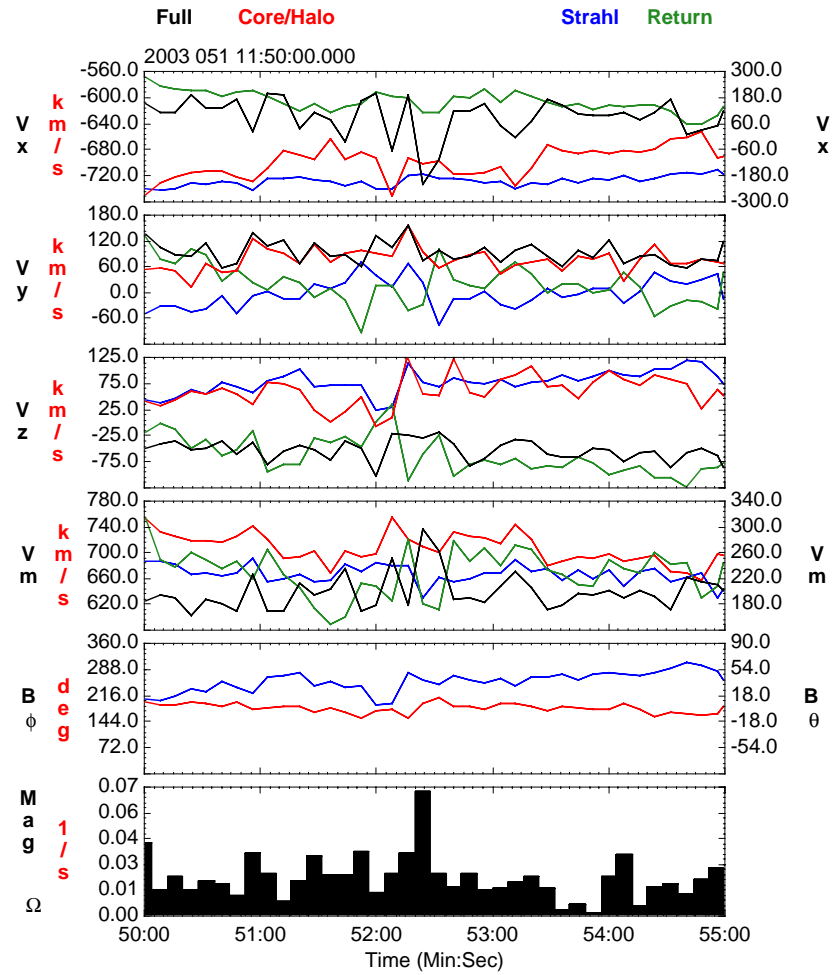

Fig. 12. Component velocities for the Full eVDF and the reduced component velocities for the Core/Halo, Strahl, and Return Population. All $m$ are from $\mathrm{C} 2$. Also shown are the $\mathrm{C} 2$ magnetic field $\varphi$ (red) and $\theta$ (blue) components and the magnitude of the vorticity. In the $V_{\mathrm{x}}$ and $V_{m}$ panels the strahl and return velocities are plotted against the right-hand axis.

electrons are the direct source of the vorticity but probably does indicate that they are the primary drivers in the overall electron response which must factor in adjustments by the core and halo populations.

What this response looks like is shown in Fig. 12. This is a rather complex set of plots which show the component velocities in GSE coordinates of all of the solar wind populations together with the velocities derived from the full eVDF. The component velocities were estimated using the method described in Sect. 7 and are shown in the plots as reduced velocities. Also shown in the figure is the total velocity of the populations $(\mathrm{Vm})$, the spherical components of the magnetic field, and the magnitude of the vorticity which is plotted as a bar chart.

The figure shows $5 \mathrm{~min}$ of data during which there are two excursions into and out of regions that contains return electrons, one lasting about $24 \mathrm{~s}$ near 12:56:30 UT and one lasting about $16 \mathrm{~s}$ near 12:57:30 UT. These are identified from the plot containing the magnitude of the velocity of the return electron population $(\mathrm{Vm}$, green trace). Recall that there is a non-zero baseline offset in the velocity of about $50 \mathrm{~km} \mathrm{~s}^{-1}$ when there are no return electrons due to background counts in the mask area. 


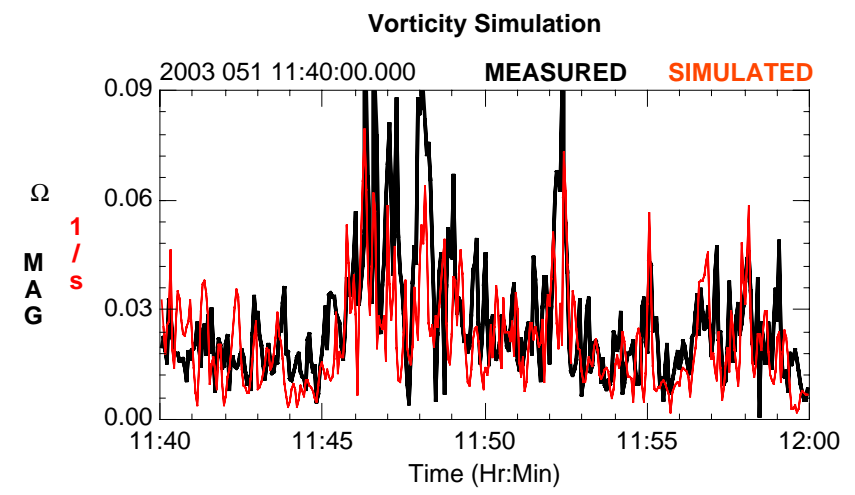

Fig. 13. Same format plot as shown in Fig. 11 but restricted to the time range shown in Fig. 8.

Both incursions into an area of return electrons are short and accompanied by jumps in the measured vorticity. The first occurs at the start of a significant rotation in the $\theta$ component of the magnetic field and the second possibly at the exit of the rotation but this is less clear. What should be noted in the plots is the response of the core/halo population to changes in the strahl and return populations. For the 12:56:30 UT event the $V_{\mathrm{x}}$ component of the return electrons remains almost unchanged while there is a significant decrease in the strahl component. This decrease is countered by an increase in the core/halo velocity which attempts to counter the decrease but as can be seen in the plot of the full eVDF component does not fully compensate for the change. The $V_{\mathrm{y}}$ component for the same time period shows a different picture. Here the changes in the return and strahl component velocities are almost equal and opposite. This causes little net overall change in $V_{\mathrm{y}}$ and because of this there is no appreciable response in the core/halo and no change in the full eVDF $V_{\mathrm{y}}$. In $V_{\mathrm{z}}$, as in $V_{\mathrm{y}}$, the return and strahl contribution to the velocity vary oppositely, however, there is a larger change in the return component than in the strahl which forces a response in the core/halo to attempt to balance out the difference. As in $V_{\mathrm{x}}$ this response is short and there is a net change in the total $V_{\mathrm{z}}$.

What is seen in Fig. 12 is that the core/halo actively works to reverse any changes in the component velocities of either the strahl and/or the return populations. That the response of the core is generally not fully successful results in small overall changes in the component velocities which are then appear as vorticity. Because many of these velocity changes are initiated by changes in the orientation of the local magnetic field is why there is such a good correlation between vorticity and the magnetic field rotations seen in Fig. 11. Figure 13 shows a higher resolution plot of the data in Fig. 11 covering the time period in Fig. 8 which gives a better indication of just how well the qualitative agreement is.

\section{Conclusions}

The electron data from the four Cluster spacecraft has provided the opportunity to compute spatial derivatives of the plasma moments in the Earth's electron foreshock. There are several questions which had to be addressed before any analysis could take place. One of the most prominent is the ability of the electron moments to adequately represent the solar wind. This was shown to be basically a non-issue. The Cluster electron velocity moments are on a par with the ion measurements where they could be compared and the density is on a par with that derived from WHISPER. The methodology used to compute both the moments and the spatial derivatives is described in detail together with associated problems.

Spatial derivatives were computed for a two and a half hour interval during which the spacecraft are in the upstream. For most of the time they are on field lines which are connected to the bow shock which is evidenced by a strong return particle signature. The vorticity in the time period exhibits increases in the vicinity of rotations in the magnetic field. Simple simulations suggest that this is due to deflections of the total velocity caused by changes in orientation of the velocity of the field-aligned strahl and reflected electron populations together with response by the core/halo to the changes. We have yet to look into whether any of these changes induce measurable changes in the ion VDF.

The coupling of the magnetic field and return particles electrons poses an interesting question: can this coupling be used to couple turbulence in the magnetic field to the particles? With only four second resolution on the plasma moments we do not have the time resolution to look at the high frequency fluctuations, however it seem entirely plausible. The comparison of turbulence in the measured vorticity and magnetic field over the frequency range available will be taken up in a future paper as will the role changes in the core/halo in the response to the overall changes in the strahl and return electrons. We also plan to look at the vorticity signatures present during the times indicated in the work by Hasegawa et al. (2006).

Acknowledgements. The authors would like to acknowledge the work and role the Cluster Active Archive (CAA) and thank the CIS, EFW, WHISPER and FGM teams for providing the data used in this study. We would also like to acknowledge the PEACE team at MSSL who worked on and are constantly improving the instrument calibration as well as the two referees for their thoughtful and excellent comments. CG would like to acknowledge support from NASA Grant NNX10AC90G.

Topical Editor R. Nakamura thanks S. Schwartz and another anonymous referee for their help in evaluating this paper. 


\section{References}

Burlaga, L. F.: A heliospheric vortex street?, J. Geophys. Res., 95, 4333-4336, 1990.

Burlaga, L. F. and Richardson, J. D.: North-south flows at 47 AU: A heliospheric vortex street?, J. Geophys. Res., 105, 10501, doi: 10.1029/1999JA000376, 2000.

Burlaga, L. F., Ness, N. F., Yang, Y. M., and Sheeley, N. R.: Heliospheric magnetic field strength and polarity from 1 to $81 \mathrm{AU}$ during the ascending phase of solar cycle 23, J. Geophys. Res., 107, 1410, doi:10.1029/2001JA009217, 2002.

Chanteur, G.: Spatial Interpolation for Four Spacecraft: Theory, in: Analysis methods for multi-spacecraft data, edited by: Paschmann, G. and Daly, P. W., p. 349, ESA Publications Division, Keplerlaan 1, 2200 AG Noordwijk, The Netherlands, 1998.

Dewhurst, J. P.: Cluster investigations of magnetotail processes, $\mathrm{PhD}$, University College London, 2005.

Fairfield, D. H., Kuznetsova, M. M., Mukai, T., Nagai, T., Gombosi, T. I., and Ridley, A. J.: Waves on the dusk flank boundary layer during very northward interplanetary magnetic field conditions: Observations and simulation, J. Geophys. Res., 112, A08206, doi:10.1029/2006JA012052, 2007.

Goldstein, M. L., Roberts, D. A., Burlaga, L. F., Siregar, E., and Deane, A. E.: North-south flows observed in the outer heliosphere at solar minimum: Vortex streets or corotating interaction regions?, J. Geophys. Res., 106, 15973, doi:10.1029/ 2000JA000106, 2001.

Gosling, J. T., Thomsen, M. F., Bame, S. J., and Russell, C. T.: Suprathermal electrons at the Earth's bow shock, J. Geophys. Res., 94, 10011-10025, 1989.

Harvey, C. C. and Schwartz, S. J.: Time Series Resampling Methods, in: Analysis methods for multi-spacecraft data, edited by: Paschmann, G. and Daly, P. W., p. 43, ESA Publications Division, Keplerlaan 1, 2200 AG Noordwijk, The Netherlands, 1998.

Hasegawa, H., Fujimoto, M., Phan, T. D., Réme, H., Balogh, A., Dunlop, M. W., Hashimoto, C., and TanDokoro, R.: Transport of solar wind into Earth's magnetosphere through rolled-up KelvinHelmholtz vortices, Nature, 430, 755-758, 2004.

Hasegawa, H., Fujimoto, M., Takagi, K., Saito, Y., Mukai, T., and Réme, H.: Single-spacecraft detection of rolled-up KelvinHelmholtz vortices at the flank magnetopause, J. Geophys. Res., 111, A09203, doi:10.1029/2006JA011728, 2006.

Henderson, P. D., Owen, C. J., Lahiff, A. D., Alexeev, I. V., Fazakerley, A. N., Lucek, E., and Réme, H.: Cluster PEACE observations of electron pressure tensor divergence in the magnetotail, Geophys. Res. Lett., 33, L22106, doi:10.1029/2006GL027868, 2006.

Henderson, P. D., Owen, C. J., Lahiff, A. D., Alexeev, I. V., Fazakerley, A. N., Yin, L., Walsh, A. P., Lucek, E., and Réme, H.: The relationship between $\mathbf{j} \times \mathbf{B}$ and $\nabla \cdot \mathbf{P}_{\mathbf{e}}$ in the magnetotail plasma sheet: Cluster observations, J. Geophys. Res., 113, A075S31, doi:10.1029/2007JA012697, 2008.

Hwang, K. J., Kuznetsova, M. M., Sahraoui, F., Goldstein, M. L., Lee, E., and Parks, G. K.: Kelvin-Helmholtz waves under southward IMF, J. Geophys. Res., submitted, 2010.

Johnstone, A. D., Alsop, C., Gurge, S., Carter, P. J., Coates, A. J., Coker, A. J., Fazakerley, A. N., Grande, M., Gowen, R. A., Gurgiolo, C., Hancock, B. K., Narheim, B., Preece, A., Sheather, P. H., Winningham, J. D., and Woodcliffe, R. D.: PEACE: A plasma electron and current experiment, Space Sci. Rev., 79,
351-398, 1997.

Lin, R. P., Larson, D. E., Ergun, R. E., McFadden, J. P., Carlson, C. E., Phan, T. D., Ashford, S., Anderson, K. A., McCarthy, M., Skoug, R., Parks, G. K., Réme, H., Bosqued, J. M., d'Uston, C., Sanderson, T. R., and Wenzel, K. P.: Observations of the solar wind, bow shock and upstream particles with the WIND 3D plasma instrument, Adv. Space. Res., 20, 645-654, 1997.

Maksimovic, M. V., Zouganelis, I., Chaufray, J. Y., Issautier, K., Scime, E. E., Littleton, J. E., Marsch, E., McComas, D. J., Salem, C., Lin, R. P., and Elliott, H.: Radial evolution of the electron distribution functions in the fast solar wind between 0.3 and $1.5 \mathrm{AU}$, J. Geophys. Res., 110, A09104, doi:10.1029/2005JA011119, 2005.

Narita, Y., Glassmeier, K. H., and Treumann, R. A.: Wave-number spectra and intermittency in the terrestrial foreshock region, Phys. Rev. Lett., 97, 191101-191104, doi:10.1103/PhysRevLett. 97.191101, 2006.

Narita, Y., Glassmeier, K. H., Sahraoui, F., Goldstein, M. L., and Treumann, R. A.: Wave-vector dependence of magneticturbulence spectra in the solar wind, Phys. Rev. Lett., 104, 171101, doi:10.1103/PhysRevLett, 104.171101, 2010.

Paschmann, G., Fazakerley, A. N., and Schwartz, S.: Moments of plasma velocity distributions, in: Analysis methods for multispacecraft data, edited by: Paschmann, G. and Daly, P. W., p. 125, ESA Publications Division, Keplerlaan 1, 2200 AG Noordwijk, The Netherlands, 1998.

Robert, P., Roux, A., Harvey, C. C., Dunlop, M. W., Daly, P. W., and Glassmeier, K.-H.: Tetrahedron Geometry Factors, in: Analysis methods for multi-spacecraft data, edited by: Paschmann, G. and Daly, P. W., p. 323, ESA Publications Division, Keplerlaan 1, 2200 AG Noordwijk, The Netherlands, 1998.

Sahraoui, F., Goldstein, M. L., Belmont, G., Canu, P., and Rezeau, L.: Three-dimensional anisotropic k-spectra of turbulence at supproton scales in the solar wind, Phys. Rev. Lett., 105, 131101131104, doi:10.1103/PhysRevLett.105.131101, 2010.

Veselovsky, I. S.: Solar wind vortex flow in the outer heliosphere, in: Physics of the Outer Heliosphere, edited by: Grzedzielski, S. and Page, D. E., p. 277, Pergamon, London, 1990.

Veselovsky, I. S. and Triskova, L.: MHD spiral vortex tubes of the solar wind in the outer heliosphere, Stud. Geophys. Geod., 34, 362-368, 1990.

Viñas, A. F. and Gurgiolo, C.: Spherical harmonic analysis of particle velocity distribution function: Comparison of moments and anisotropies using Cluster data, J. Geophys. Res., 114, A01105, doi:10.1029/2008JA013633, 2009.

Vogt, J. and Paschmann, G.: Accuracy of plasma moment derivatives, in: Analysis methods for multi-spacecraft data, edited by: Paschmann, G. and Daly, P. W., p. 419, ESA Publications Division, Keplerlaan 1, 2200 AG Noordwijk, The Netherlands, 1998.

Volpp, J. and Sieg, D.: ESOC data products in the CAA, in: The Cluster Active Archive, edited by: Laakso, H., Taylor, M., and Escoubet, C. P., Springer, London, England., 2010.

Wu, C. S.: A fast Fermi process: Energetic electrons accelerated by a nearly perpendicular bow shock, J. Geophys. Res., 89, 88578862, 1984. 